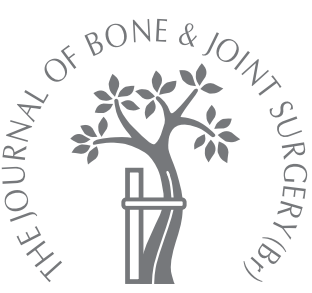

K. Goto, K. Kawanabe, H. Akiyama, T. Morimoto, T. Nakamura

From Kyoto University, Kyoto, Japan

\title{
Clinical and radiological evaluation of revision hip arthroplasty using the cement-in- cement technique
}

\begin{abstract}
We reviewed 44 consecutive revision hip replacements in 38 patients performed using the cement-in-cement technique. All were performed for acetabular loosening in the presence of a well-fixed femoral component. The mean follow-up was 5.1 years (2 to 10.1).

Radiological analysis at final follow-up indicated no loosening of the femoral component, except for one case with a continuous radiolucent line in all zones and peri-prosthetic fracture which required further revision. Peri-operative complications included nine proximal femoral fractures $(20.4 \%)$ and perforation of the proximal femur in one hip. In five hips wiring or fixation with a braided suture was undertaken but no additional augmentation was required. There was an improvement in the mean Japanese Orthopaedic Association score from 55.5 ( 28 to 81 ) pre-operatively to 77.8 (40 to 95$)$ at final follow-up ( $<<0.001$ ). Revision using a cement-in-cement technique allows increased exposure for acetabular revision and is effective in the medium term. Further follow-up is required to assess the long-term results in the light of in vitro studies which have questioned the quality of the cement-in-cement bond.
\end{abstract}

Aseptic loosening is a major cause of failure in total hip replacement (THR), ${ }^{1-3}$ and in many cases an acetabular component is loose in the presence of a well-fixed cemented femoral component. ${ }^{4}$ Revision of the acetabular component is difficult and often requires wide exposure of the acetabulum if the femoral component is left in place. In such cases, trochanteric osteotomy or extensive soft-tissue mobilisation with anterior or posterior translation of the proximal femur are often considered. However, trochanteric osteotomy may jeopardise the intact bone-cement interface and cause nonunion of the greater trochanter, ${ }^{5}$ and extensive soft-tissue releasing can damage neurovascular structures and cause instability of the reconstructed hip joint. ${ }^{4}$ Correction of leg-length discrepancy or a gain in optimal joint tension to avoid dislocation can prove difficult when extensive acetabular reconstruction is performed in revision surgery with the femoral component left in place. Therefore, simultaneous revision of a loose acetabular component and wellfixed femoral component is often considered. In addition, revision of a well-fixed femoral component should be considered when recurrent dislocation occurs due to malpositioning of the component, or there has been proximal stem breakage. ${ }^{6,7}$
The cement-in-cement technique provides an option for revision of a well-fixed cemented stem; this method was first described by Eftekhar ${ }^{8}$ in 1978 and validated experimentally by Greenwald, Narten and Wilde, ${ }^{9}$ with clinical results reported by several authors. ${ }^{6,7,10,11}$ However, the use and indications of this technique are not commonly known, presumably in part because no detailed radiological evaluation has been reported. Therefore, the purpose of this study was to review a series of revision THRs using the re-cementing technique, focusing on radiological changes at the cement-bone interface.

\section{Patients and Methods}

Between April 1996 and June 2004, 44 consecutive revision THRs in 38 patients were performed by several orthopaedic surgeons (including KK) using the cement-in-cement technique in our hospital. All the patients were followed up for more than two years and reviewed retrospectively. There were 37 women and one man with a mean age of 66 years (48 to 78$)$, mean weight of $52.3 \mathrm{~kg}$ (33 to 67), and mean body mass index (BMI) of $24.0 \mathrm{~kg} / \mathrm{m}^{2}$ (16.6 to 31 ) at the time of revision. The original diagnosis was primary or secondary osteoarthritis in all patients, and the mean time from the initial to the revision sur- 
gery was 15.3 years (7.9 to 30.2). A total of 40 THRs were revised for loosening of the acetabular component and four bipolar hemiarthroplasties were revised for penetration of the bipolar head. The femoral components which were revised comprised 26 Charnley components (Thackray, Leeds, United Kingdom), ten Bioceram (Kyocera, Kyoto, Japan), seven KC (Kyocera) and one unknown type.

Revision operations were performed through a direct lateral approach (26 Hardinge ${ }^{12}$ and 18 Dall approaches ${ }^{13}$ ). In 41 hips a transtrochanteric approach had been undertaken at the primary surgery. In each of these cases the trochanteric wires were removed before proceeding with the revision. The cement-bone and cement-stem interfaces in the proximal femoral portion were carefully identified and the original cement mantle at the stem shoulder was meticulously curetted out. The femoral component was then carefully hammered out to avoid introducing torsional movement that could lead to damage to the rest of the original cement mantle. The proximal femur was retracted distally and posteriorly, and the acetabular component was revised with wide exposure. Subsequently, the proximal femoral cement mantle in zones 1 or 7 , as described by Gruen, McNeice and Amstutz, ${ }^{14}$ and the underlying fibrous or osteolytic granulation tissue were curetted out thoroughly to a depth where cement-bone contact could be visually confirmed. Neocortex in the proximal femur ${ }^{15}$ was removed with a high-speed burr, and any disuse osteoporotic lesions formed by stress shielding outside the neocortex were also curetted out. We then attempted to insert a femoral component of a similar shape and size (or marginally smaller) to those of the retrieved component. The inner surface of the distal cement mantle was roughened with a highspeed burr, and the internal cavity of the cement mantle was carefully enlarged with the burr if insertion of the new component into the original position proved difficult. After lavage to wash out debris and clots, the cement mantle cavity was dried with gauze, and polymethylmethacrylate (PMMA) bone cement of relatively low viscosity was injected with a gun prior to insertion of the new femoral component.

The new femoral components were PHS (physio-hip-system) type 6 (30), KC (seven), PHS type 7 (three), and HS-3 (four), all produced in titanium alloy by Japan Medical Materials, Osaka, Japan. The modular heads (Japan Medical Materials) were $22 \mathrm{~mm}$ alumina in seven cases, $22 \mathrm{~mm}$ zirconia in 27 cases, and $26 \mathrm{~mm}$ zirconia in ten cases. Using the cement-in-cement technique, CMW 3 bone cement (DePuy, Blackpool, United Kingdom) was used in 12 hips and Endurance bone cement (DePuy) in the remainder. For revision of the acetabular component, reconstruction was performed using a Kerboul acetabular reinforcement device (Howmedica, Hérouville, France) in eight hips and a KT plate (Japan Medical Materials) in 33. In total 43 PHS acetabular components (Japan Medical Materials) were used and one CLHO acetabular component (Japan Medical

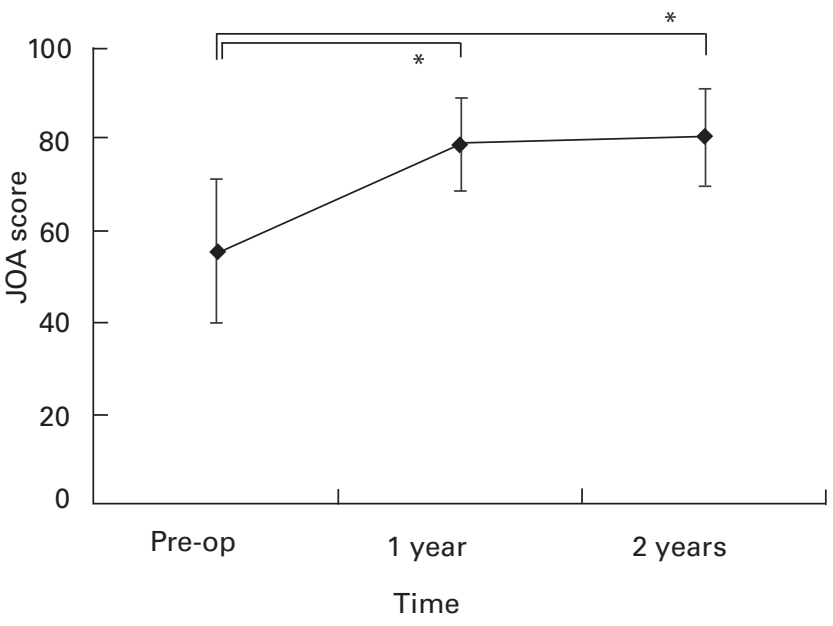

Fig. 1

Mean Japanese Orthopaedic Association (JOA) scores pre-operatively and at one and two years post-operatively. The error bars indicate standard deviation. Statistical significance was found between the preoperative and post-operative scores at each interval ( ${ }^{*}$ ANOVA, $p<0.001$ ).

Materials). The mean operation time was 210 minutes (141 to 271 ) and the mean blood loss during surgery was $661 \mathrm{~g}$ (240 to 1440).

The mean follow-up period was 5.1 years (2 to 10.1). Standard anteroposterior radiographs of the pelvis were taken immediately post-operatively, at two, four, six and eight weeks and three, six, nine and 12 months, and sixmonthly or annually thereafter. Radiological loosening was assessed according to the criteria of Harris, McCarthy and O'Neill ${ }^{16}$ with peri-prosthetic radiolucencies evaluated in the Gruen zones. The presence of heterotopic ossification was recorded using the Brooker classification. ${ }^{17}$ Hip function was evaluated using the Japanese Orthopaedic Association (JOA) score, ${ }^{18}$ which is based on pain $(40 \%)$, range of movement $(20 \%)$, ability to walk $(20 \%)$ and activities of daily living (ADL) (20\%). The total score is 100 points for a normal hip. Peri- and post-operative complications were identified from the medical records.

Statistical analysis. Repeated analysis of variance (ANOVA) was used to compare the pre-operative and follow-up JOA score. Kaplan-Meier analysis with $95 \%$ confidence intervals (CI) was performed to evaluate the period to the appearance of a radiolucent line exceeding $1 \mathrm{~mm}$ in width. Log-rank testing was undertaken to test the relationship between possible risk factors and radiolucent line-free survival. The possible risk factors included age, BMI, body weight, pre-operative JOA score, type of femoral component, surgical approach, pre-operative presence of radiolucent lines, and peri-operative fractures. In log-rank tests for the first four of these variables, the cases were divided into two groups based on the mean value of each variable. All statistical analyses were carried out using JMP IN software version 5.1.2 (SAS Institute 


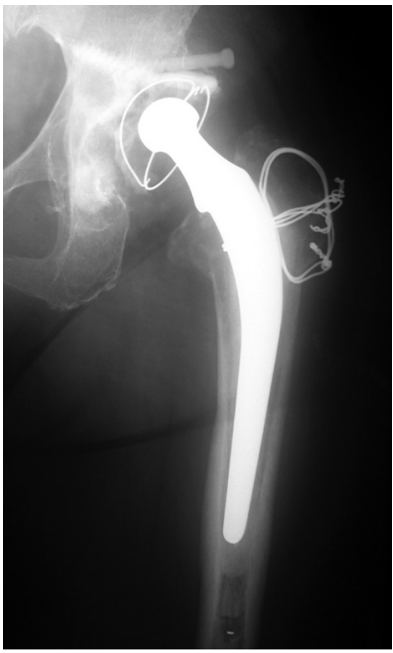

Fig. 2a

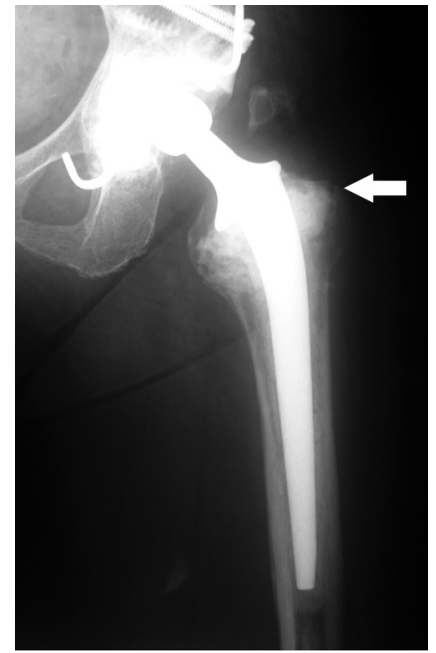

Fig. 2b

Radiographs of a 66-year-old woman with acetabular loosening who had revision surgery 15.4 years after primary hip replacement. a) Preoperative anteroposterior (AP) radiograph, in which no femoral radiolucent line was found but stress shielding was evident, and b) AP radiograph 5.6 years after revision, showing a radiolucent line in Gruen zone 1 (arrow).

Inc., Cary, North Carolina) and SAS software version 9.1 (SAS Institute Inc). Two-sided p-values $<0.05$ were considered statistically significant.

\section{Results}

Peri-operative fractures occurred in ten cases $(22.7 \%)$, involving the greater trochanter or proximal femur in nine $(20.9 \%)$, of which five required fixation; with wiring in four hips $(20.4 \%)$ and braided sutures in one. In two hips trochanteric fractures were left without fixation; producing asymptomatic pseudarthroses. In one hip perforation of the distal femur resulted in an extensive cement extrusion, which was removed intra-operatively and required no further treatment. Early post-operative complications included dislocation in three hips $(6.8 \%)$, which required closed reduction, and one recurrent dislocation which became stable by the end of the first post-operative year. Class 3 heterotopic ossification ${ }^{17}$ developed in one hip, but there were no associated symptoms, and there were no cases with peri-prosthetic infection or deep-vein thrombosis. The mean JOA score improved from 55.5 (28 to 81) pre-operatively to 78.8 ( 57 to 93 ) at one year post-operatively, 80.4 (55 to 96) at two years post-operatively, and 77.8 (40 to 95 ) at final follow-up (Fig. 1). The differences between the pre-operative and one- and two-year postoperative scores were statistically significant (ANOVA, $\mathrm{p}<$ $0.001)$.

Radiological analysis at final follow-up, according to the criteria of Harris et al, ${ }^{16}$ indicated no definite, probable or possible loosening of the femoral component (Fig. 2), except in one hip with possible radiological loosening and

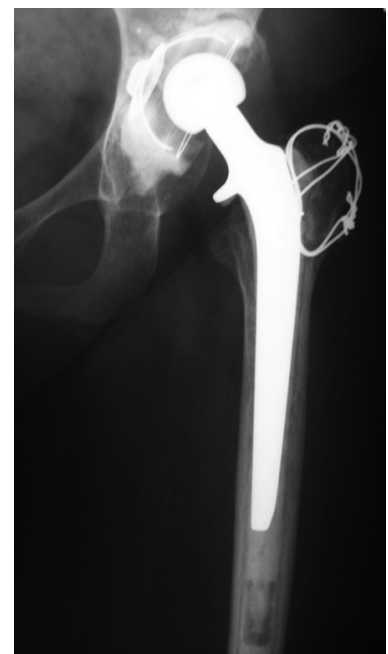

Fig. $3 a$

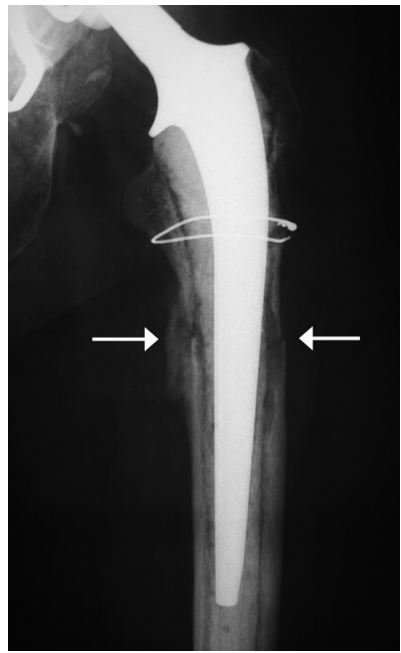

Fig. 3b
Radiographs of a 56-year-old woman with acetabular loosening who had revision surgery 9.9 years after primary hip replacement. a) Preoperative anteroposterior (AP) radiograph, in which a radiolucent line in zones 1, 2 and 7 was evident and b) AP radiograph seven years after the revision and immediately prior to further revision. The radiolucent line had extended to all zones, with widening and osteolysis in zones 2 and 6 where a proximal femoral fracture was confirmed (arrows).

peri-prosthetic fracture (Fig. 3). This was in a 56-year-old woman who required a further revision with strut allograft augmentation seven years after the initial revision surgery. There were no other radiolucent lines or osteolysis beyond zone 1 or 7 in any hip. Changes in the extent of the pre- and post-operative radiolucent lines and radiological osteolysis are summarised in Tables I to III.

Kaplan-Meier analysis using the appearance of a radiolucent line $1 \mathrm{~mm}$ in width in any zone as the endpoint indicated survival rates of $71.5 \%$ (95\% CI 52.3 to 84.1$)$ at five years and $53.5 \%$ (95\% CI 30.2 to 72.1 ) at ten years (Fig. 4). Log-rank tests showed that all the possible risk factors examined had no significant influence on the appearance of a radiolucent line $>1 \mathrm{~mm}$ in any area (Table IV).

\section{Discussion}

In our series 18 hips $(41 \%)$ had radiolucent lines or osteolysis confined to Gruen zones 1 and 7 pre-operatively. These proximal gaps between the cement and bone must be regarded as a potential pathway for wear debris, which is thought to initiate osteolysis. ${ }^{19-22}$ In our single hip requiring further revision, the pre-operative radiolucent line could still be identified post-operatively, indicating that removal of the fibrous membrane or sclerotic neocortex ${ }^{15}$ with underlying osteoporotic regions had been inadequate. Lieberman ${ }^{23}$ suggested that the neocortex must be removed when performing a cemented femoral revision to obtain adequate interdigitation between the new cement and the bone. Removal of part of the proximal cement mantle is necessary to permit the safe extraction of the original component, especially for a proximally curved device such as a 
Table I. Hips with a radiolucent line greater than $1 \mathrm{~mm}$ or osteolysis preceding revision and at final follow-up

\begin{tabular}{lcc}
\hline & Preceding revision (\%) & Final follow-up (\%) \\
\hline Osteolysis & $14(31.8)$ & $2(4.5)^{*}$ \\
Radiolucent line & $5(11.4)$ & $13(29.5)^{*}$ \\
\hline * including the revision case & &
\end{tabular}

* including the revision case

Table II. Distribution of radiolucent lines greater than $1 \mathrm{~mm}$ or osteolysis present. The number of hips with a radiolucent line present in the same zone pre-operatively as postoperatively at final follow-up is shown in parentheses

\begin{tabular}{lll}
\hline & Preceding revision $(\mathbf{n}=\mathbf{1 9})$ & Final follow-up $(\mathbf{n}=\mathbf{1 4})$ \\
\hline Zone 1 or 7 , or 1 and 7 & 18 & $13(10)$ \\
Beyond zones 1 and 7 & $1^{*}$ & $1^{*}$ \\
\hline
\end{tabular}

* revision case

Table III. Time post-operatively at which a radiolucent line greater than $1 \mathrm{~mm}$ was first identified

\begin{tabular}{ll}
\hline Time & Number of cases \\
\hline Immediately post-operatively & 3 of $14^{*}$ \\
Less than three years & 6 of 14 \\
Over three years & 5 of 9 \\
\hline * including the revision case &
\end{tabular}

Charnley prosthesis, and to confirm that the bone-cement interface is intact. In our single hip requiring further revision it is also possible that the radiolucent lines might have extended to the distal femur, because the lateral radiolucent line was found to reach zone 2 on the pre-operative radiograph. In such cases, visual confirmation of the distal bonecement interface is hard to achieve. The cement-in-cement technique should probably not be undertaken where a radiolucency extends beyond zones 1 or 7 .

Kaplan-Meier analysis estimated a survival rate of $53.5 \%$ for the appearance of a radiolucent line $>1 \mathrm{~mm}$ in width in any area at ten years, but a post-operative radiolucent line in excess of $1 \mathrm{~mm}$ was not identified beyond zone 1 or 7 in any hips except the revision case. This suggests that removing the femoral component and recementing it in the old cement mantle did not have a detrimental effect, and this supports the use of the cement-in-cement technique. The incidence of peri-operative femoral fractures in our series was high, occurring in nine hips $(20.4 \%)$, which is greater than described in previous reports of cemented revisions. ${ }^{24,25}$ The removal of a well-fixed femoral component is itself clearly a risk for femoral fracture compared with removal of a loosened stem. The removal of the trochanteric wires required in 41 of our hips (93\%) may have weakened the greater trochanter and contributed to

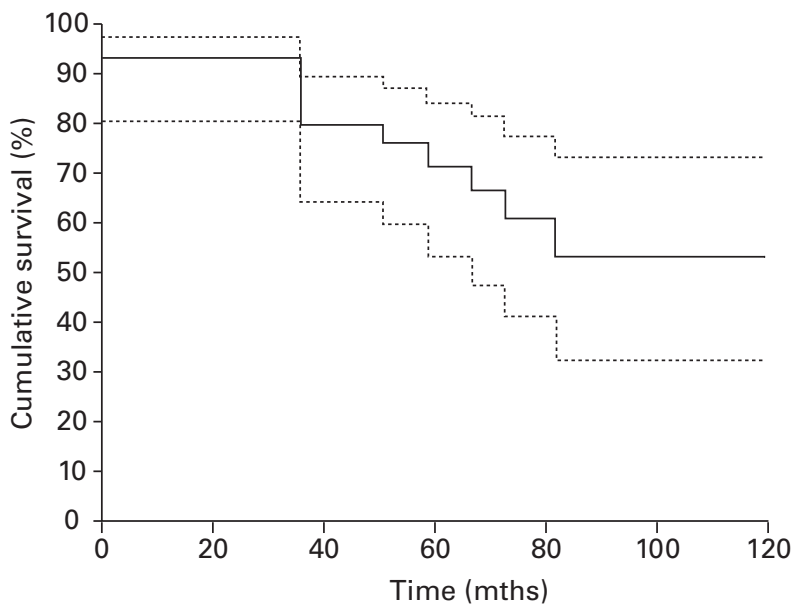

Fig. 4

Kaplan-Meier analysis using the appearance of a femoral radiolucent line wider than $1 \mathrm{~mm}$ in any area as the endpoint. The dotted lines indicate $95 \%$ confidence intervals.

the subsequent fractures. None of the fractures required additional treatment other than wiring.

In order to revise a loose acetabular component with a well-fixed, cemented femoral component, complete removal of cement and insertion of a new stem is often advised in order to avoid future femoral problems arising from older cement. However, removal of a distal cement mantle with an intact bone-cement interface is timeconsuming and risks femoral fracture and perforation. ${ }^{26-28}$ A perforation rate as high as $16 \%$ has been reported, ${ }^{26,27}$ but we only encountered this problem once. In an in vitro study, ${ }^{29}$ the shear strength of the cement-bone interface has been shown to diminish to $20.6 \%$ of its initial value with 
Table IV. Variables examined as possible risk factors using log-rank tests

\begin{tabular}{|c|c|c|}
\hline Variable $^{*}$ & Value & p-value \\
\hline Mean age at surgery in yrs (range) ${ }^{\dagger}$ & 66 (48 to 78$)$ & 0.79 \\
\hline Mean body weight in kg $(\text { range })^{\dagger}$ & 52.3 (33 to 67$)$ & 0.53 \\
\hline Mean BMI in $\mathrm{kg} / \mathrm{m}^{2}$ (range) ${ }^{\dagger}$ & $24.0(16.6$ to 31$)$ & 0.82 \\
\hline Pre-operative JOA score ${ }^{\dagger}$ & $55.5(28$ to 81$)$ & 0.51 \\
\hline \multicolumn{3}{|l|}{ Surgical approach } \\
\hline Hardinge & 26 & 0.27 \\
\hline Dall & 18 & \\
\hline \multicolumn{3}{|l|}{ Applied femoral prostheses } \\
\hline PHS type 6 & 30 & 0.73 \\
\hline $\mathrm{KC}$ & 7 & 0.29 \\
\hline PHS type 7 & 3 & 0.20 \\
\hline HS-3 & 4 & 0.80 \\
\hline \multicolumn{3}{|l|}{ Fracture or crack during surgery } \\
\hline Greater trochanter & 7 & 0.36 \\
\hline Proximal femoral crack & 2 & 0.66 \\
\hline \multicolumn{3}{|c|}{ Lucent line over $1 \mathrm{~mm}$ or osteolysis before surgery } \\
\hline Yes & 19 & 0.21 \\
\hline No & 25 & \\
\hline
\end{tabular}

the first revision and to $6.8 \%$ on the second revision. In contrast, good clinical results have been reported with the cement-in-cement technique, ${ }^{30}$ which were comparable to the results we obtained, although previous reports have included less radiological information. $6,7,10$

In order to increase the shear strength between old and new cement, the inner surface of the old cement requires roughening. Greenwald et $\mathrm{al}^{9}$ evaluated the cement-incement technique in vitro and showed that where the old cement surface was rasped and dried, the shear strength between old and new cement was reduced by only $6 \%$, compared with the strength of uniform cement specimens. However, in practice, we found it difficult to roughen all the inner surface of the old cement mantle to a uniform extent, because the whole of the mantle is difficult to visualise. This sometimes results in the unintentional creation of a pit or defect in the mantle, which is seen on postoperative radiographs as enlargement of the mantle or cement leakage. These defects did not seem to have a detrimental effect on the cement mantle or the clinical results. However, Li, Ingle and Dowell ${ }^{31}$ showed in vitro that the presence of a thin layer of blood and marrow debris at the interface weakens the cement-to-cement bond by $80 \%$ to $85 \%$. Accordingly, our patients require careful follow-up to determine whether any interface defects will emerge.

An application for cement-in-cement technique includes recurrent dislocation due to malposition of the femoral component, and proximal breakage of the femoral component with an intact distal cement mantle. ${ }^{6,7}$ All the femoral revisions in our series were performed to increase exposure of the acetabulum, or because of a mismatch between an old femoral component and the intended revision acetabular component. Good clinical results were obtained in all but one hip at a mean follow-up of 5.1 years.

No long-term follow-up of revision cases using the cement-in-cement technique has been reported, and there are only a few studies of the mechanical aspects of this technique. ${ }^{9,31,32}$ However, our mid-term results suggest that this technique is appropriate for revision surgery in selected cases.

No benefits in any form have been received or will be received from a commercial party related directly or indirectly to the subject of this article.

\section{References}

1. Herberts $\mathbf{P}$, Malchau $\mathbf{H}$. Long-term registration has improved the quality of hip replacement: a review of the Swedish THR Register comparing 160,000 cases. Acta Orthop Scand 2000;71:111-21.

2. Lucht U. The Danish Hip Arthroplasty Register. Acta Orthop Scand 2000;71:433-9.

3. Puolakka TJ, Pajamäki KJ, Halonen PJ, et al. The Finnish Arthroplasty Register: report of the hip register. Acta Orthop Scand 2001;72:433-41.

4. Nabors ED, Liebelt R, Mattingly DA, Bierbaum BE. Removal and reinsertion of cemented femoral components during acetabular revision. J Arthroplasty 1996;11:146-52.

5. Amstutz HC, Ma SM, Jinnah RH, Mai L. Revision of aseptic loose total hip arthroplasties. Clin Orthop 1982;170:21-33.

6. Lieberman JR, Moeckel BH, Evans BG, Salvati EA, Ranawat CS. Cementwithin-cement revision hip arthroplasty. J Bone Joint Surg [Br] 1993;75-B:869-71.

7. Quinlan JF, O'Shea K. Doyle F, Brady OH. In-cement technique for revision hip arthroplasty. J Bone Joint Surg [Br] 2006;88-B:730-3.

8. Eftekhar NS. Principles of total hip arthroplasty. St Louis: Mosby, 1978.

9. Greenwald AS, Narten NC, Wilde AH. Points in the technique of recementing in the revision of an implant arthroplasty. J Bone Joint Surg [Br] 1978;60-B:107-10.

10. McCallum JD 3rd, Hozack WJ. Recementing a femoral component into a stable cement mantle using ultrasonic tools. Clin Orthop 1995;319:232-7. 
11. Mandziak DG, Howie DW, Neale SD, McGee MA. Cement-within-cement stem exchange using the collarless polished double-taper stem. J Arthroplasty 2007:22:1000-6

12. Hardinge K. The direct lateral approach to the hip. J Bone Joint Surg [Br] 1982;64B:17-19.

13. Dall D. Exposure of the hip by anterior osteotomy of the greater trochanter: a modified anterolateral approach. J Bone Joint Surg [Br] 1986;68-B:382-6.

14. Gruen TA, McNeice GM, Amstutz HC. "Modes of failure" of cemented stem-type femoral components: a radiographic analysis of loosening. Clin Orthop 1979;141:1727.

15. Harris WH. Will stress shielding limit the longevity of cemented femoral components of total hip replacement? Clin Orthop 1992;274:120-3.

16. Harris WH, McCarthy JC Jr, O'Neill DA. Femoral component loosening using contemporary techniques of femoral cement fixation. J Bone Joint Surg [Am] 1982;64A:1063-7.

17. Brooker AF, Bowerman JW, Robinson RA, Riley LH Jr. Ectopic ossification following total hip replacement: incidence and a method of classification. J Bone Joint Surg [Am] 1973;55-A:1629-32

18. Mibe J, Imakiire A, Watanabe T, Fujie T. Results of total hip arthroplasty with bone graft and support ring for protrusio acetabuli in rhematoid arthritis. J Orthop Sci 2005;10:8-14

19. Salvati EA, Betts F, Doty SB. Particulate metallic debris in cemented total hip arthroplasty. Clin Orthop 1993;293:160-73.

20. Santavirta S, Hoikka V, Eskola A, et al. Aggressive granulomatous lesions in cementless total hip arthroplasty. J Bone Joint Surg [Br] 1990;72-B:980-4.

21. Goldring SR, Jasty M, Roelke MS, et al. Formation of a synovial-like membrane at the bone-cement interface: its role in bone resorption and implant loosening after total hip replacement. Arthritis Rheum 1986;29:836-42.
22. Rahbek O, Overgaard S, Lind M, et al. Sealing effect of hydroxyapatite coating on peri-implant migration of particles: an experimental study in dogs. J Bone Joint Surg [Br] 2001;83-B:441-7.

23. Lieberman JR. Cemented femoral revision: lest we forget. I Arthroplasty 2005;20(Suppl 2):72-4

24. Davis CM 3rd, Berry DJ, Harmsen WS. Cemented revision of failed uncemented femoral components of total hip arthroplasty. J Bone Joint Surg [Am] 2003;85A:1264-9.

25. Farfalli GL, Buttaro MA, Piccaluga F. Femoral fractures in revision hip surgeries with impacted bone allograft. Clin Orthop 2007;462:130-6.

26. Turner RH, Mattingly DA, Scheller A. Femoral revision total hip arthroplasty using a long-stem femoral component: clinical and radiographic analysis. J Arthroplasty 1987;2:247-58.

27. Kershaw CJ, Atkins RM, Dodd CA, Bulstrode CJ. Revision total hip arthroplasty for aseptic failure: a review of 276 cases. J Bone Joint Surg [Br] 1991;73-B:564-8.

28. Christensen CM, Seger BM, Schultz RB. Management of intraoperative femur fractures associated with revision hip arthroplasty. Clin Orthop 1989;248:177-80.

29. Dohmae Y, Bechtold JE, Sherman RE, Puno RM, Gustilo RB. Reduction in cement-bone interface shear strength between primary and revision arthroplasty. Clin Orthop 1998;236:214-20

30. Keeling P, Prendergast PJ, Lennon AB, Kenny PJ. Cement-in-cement revision hip arthroplasty: an analysis of clinical and biomechanical literature. Arch Orthop Trauma Surg 2007 [Epub ahead of print].

31. Li PI, Ingle PJ, Dowell JK. Cement-within-cement revision hip arthroplasty: should it be done? J Bone Joint Surg [Br] 1996;78-B:809-11.

32. Weinrauch PC, Bell C, Wilson L, et al. Shear properties of bilaminar polymethylmethacrylate cement mantles in revision hip joint arthroplasty. J Arthroplasty 2007;22:394-403. 